\title{
Potential of Preoperative Voiding Efficiency in Predicting Postoperative A-1 Adrenergic Antagonist Usage After Transurethral Resection of the Prostate
}

\section{Wei-Ming Cheng}

Taipei City Hospital

\section{Yi-Chun Chiu}

Taipei City Hospital

\section{Yu-Hua Fan}

Taipei Veterans General Hospital

Che-Wei Hsu

Taipei City Hospital

Chan-Chi Chang

Taipei City Hospital

Shih-Han Weng

Taipei City Hospital

Te-Wei Chang ( $\sim$ Urotwchang@gmail.com )

Taipei City Hospital

\section{Research Article}

Keywords: a-1 adrenergic antagonists, benign prostatic obstruction, transurethral resection

Posted Date: January 14th, 2021

DOl: https://doi.org/10.21203/rs.3.rs-144010/v1

License: (9) (i) This work is licensed under a Creative Commons Attribution 4.0 International License. Read Full License 


\section{Abstract}

Background: Transurethral resection of the prostate (TURP) is the treatment of choice for patients with benign prostatic obstruction (BPO) who are refractory to medical treatment. Nevertheless, some patients still need a-1 adrenergic antagonists to control their post-TURP lower urinary tract symptoms (LUTS).

Methods: In the present study, we developed a preoperative predictor of the continuous usage of a-1 adrenergic antagonists for post-TURP LUTS. We consecutively enrolled patients who underwent TURP at our hospital and divided them based on their use of a-1 adrenergic antagonists for more than 3 months postoperatively. Preoperative demographic data, including urodynamic parameters, were compared between the two groups. Forty-one patients were included in the study, with 17 patients (41\%) receiving long-term a-1 adrenergic antagonists for their post-TURP LUTS.

Results: Only lower pre-TURP voiding efficiency was significantly associated with long-term post-TURP $\mathrm{a}-1$ adrenergic antagonist usage $(63.3 \%$ vs. $75.1 \%, p=0.03)$. A pre-TURP voiding efficiency of less than $75.48 \%$ had the greatest predictive ability, with a sensitivity of $88 \%$ and specificity of $50 \%$.

Conclusions: A preoperative voiding efficiency of less than $75.48 \%$ is a simple and noninvasive predictive factor for LUTS and would be helpful in preoperative evaluation and patient counseling.

\section{Background}

Benign prostatic obstruction (BPO) is a common disease among the elderly, affecting approximately half of the men between 51 and 60 years. ${ }^{1-3}$ The disease contributes to not only lower urinary tract symptoms (LUTS) but also complications, such as the formation of vesical stones and impaired renal function. ${ }^{4}$ Transurethral resection of the prostate (TURP) is the treatment of choice for patients with BPO with complications. It is also effective to improve LUTS refractory to medical treatment; however, some patients still need a-1 adrenergic antagonists to control their post-TURP LUTS, which may impair their postoperative satisfaction. It is therefore crucial to predict the use of a-1 adrenergic antagonists after TURP in preoperative evaluation and patient counseling. In the present study, we aimed to develop a preoperative predictor for post-TURP LUTS, which needs continuous usage of a-1 adrenergic antagonists.

\section{Methods}

We retrospectively collected data on consecutive patients who underwent TURP at our hospital from January 1, 2011, to December 31, 2016. Those with incomplete preoperative evaluation, prostate malignancy, laser prostatectomy, and loss of follow-up postoperatively were excluded. The included patients were divided based on whether they needed a-1 adrenergic antagonists for more than 3 months postoperatively. Demographic data including age, voided volume, maximal flow rate (Qmax) and mean flow rate on preoperative uroflowmetry, post-void residual urine (PVR), preoperative serum prostate specific antigen, and prostate size estimated using transrectal ultrasound within 3 months before the surgical intervention were obtained. The weight of the resected prostatic tissues was also measured. 
Voiding efficiency is the percentage of the voided volume compared with the total bladder volume. The Fisher exact test and Mann-Whitney $U$ test were used to identify the possible predictors of post-TURP a-1 adrenergic antagonist usage. A receiver operating characteristic $(\mathrm{ROC})$ curve was generated to show the accuracy of the predictor with the area under curve (AUC) and determine the most suitable cutoff point by the highest sum of the sensitivity and specificity. Statistical analyses were performed using the Statistical Analysis Software, Version 9.4 (SAS Institute Inc., Cary, NC, USA). A $p$ value of $<0.05$ was considered statistically significant.

\section{Results}

Forty-one patients were included in the present study. Seventeen patients (41\%) had post-TURP LUTS, which required long-term a-1 adrenergic antagonist usage, while the other $24(59 \%)$ did not require it. The demographic data of these two groups of patients are shown in Table 1. Only lower voiding efficiency was significantly associated with long-term post-TURP a- 1 adrenergic antagonist usage $(63.3 \%$ vs. $75.1 \%$, $p=0.03$ ). The ROC curve of voiding efficiency is presented in Fig. 1 . The AUC was 0.710 , and the most suitable cutoff point was $75.48 \%$. This cutoff point had a sensitivity of $88 \%$ and specificity of $50 \%$. 
Table 1

Demographics of patients with and without long-term a-1 adrenergic antagonist usage after transurethral resection of the prostate

\begin{tabular}{|c|c|c|c|}
\hline & \multicolumn{2}{|c|}{$\begin{array}{l}\text { a-1 adrenergic antagonist usage after } \\
\text { TURP }\end{array}$} & \multirow[t]{3}{*}{$\begin{array}{l}p \\
\text { value }\end{array}$} \\
\hline & No & Yes & \\
\hline & $(n=24)$ & $(n=17)$ & \\
\hline \multicolumn{3}{|l|}{ Age (years) } & \multirow[t]{3}{*}{0.279} \\
\hline$<70$ & $14(58.33)$ & $7(41.18)$ & \\
\hline$\geq 70$ & $10(41.67)$ & $10(58.82)$ & \\
\hline Voiding efficiency (\%) & $\begin{array}{l}75.14(38.31- \\
98.63)\end{array}$ & $\begin{array}{l}63.29(30.11- \\
88.97)\end{array}$ & $0.030 *$ \\
\hline Voided volume (mL) & $179(77-314)$ & $169(56-336)$ & 0.257 \\
\hline Total volume (mL) & $243(152-464)$ & $254(175-458)$ & 0.989 \\
\hline Qmax (mL/s) & $9.75(2.50-33.90)$ & $8.90(3.40-20.20)$ & 0.674 \\
\hline Qmean (mL/s) & $4.20(0.80-18.70)$ & $4.00(1.40-7.90)$ & 0.455 \\
\hline PSA (ng/mL) & $4.73(1.30-50.11)$ & $7.38(1.35-17.29)$ & 0.645 \\
\hline $\begin{array}{l}\text { Prostate size estimated by transrectal } \\
\text { ultrasound }\left(\mathrm{cm}^{3}\right)\end{array}$ & $\begin{array}{l}60.95(30.00- \\
127.00)\end{array}$ & $\begin{array}{l}66.00(39.00- \\
125.00)\end{array}$ & 0.373 \\
\hline Resected prostate (g) & $\begin{array}{l}15.50(2.50- \\
56.00)\end{array}$ & $\begin{array}{l}15.00(1.80- \\
63.00)\end{array}$ & 0.732 \\
\hline \multicolumn{4}{|c|}{${ }^{\star} p<0.05$. All data are presented as "median (min-max)" except age. } \\
\hline $\begin{array}{l}\text { PSA, prostate-specific antigen; Qmax } \\
\text { uroflowmetry; TURP, transurethral res }\end{array}$ & $\begin{array}{l}\text { rate on uroflown } \\
\text { rostate }\end{array}$ & Qmean, mean fl & te on \\
\hline
\end{tabular}

\section{Discussion}

In the present study, we observed that the prevalence of post-TURP LUTS is not rare; moreover, a preoperative voiding efficiency of less than $75.48 \%$ could predict the postoperative medication with a-1 adrenergic antagonists. The findings of the present study could be helpful in preoperative patient counseling.

Post-TURP LUTS is a bothersome situation for patients, resulting in prolonged medication postoperatively. ${ }^{5} \mathrm{~A}$ possible pathophysiology of persistent LUTS after TURP is due to residual prostatic tissue or bladder neck dysfunction. Another possible pathophysiology is chronic bladder outlet resistance due to prostatic obstruction. ${ }^{6-8}$ This chronic resistance induces detrusor muscle hypertrophy over time, 
leading to a low compliance and small capacity of the urinary bladder. ${ }^{9}$ Urinary bladder distention due to chronic obstruction also results in ischemic injury to the urinary bladder, which in turn causes bladder wall thickening. ${ }^{10}$ These changes happen not only at a macrolevel but also at a molecular level. ${ }^{11}$ Collagen types I and III of the detrusor muscle become upregulated at the transcriptional level. Muscarinic receptors CHRM2 and CHRM3 also become overexpressed, leading to detrusor overactivity. The progression may be reversed by TURP at the early stage but not in the late stage. ${ }^{5}$

Post-TURP LUTS involves storage and voiding symptoms, both of which could be treated with a-1 adrenergic antagonists. ${ }^{12}$ These antagonists could inhibit a-1 adrenergic receptors (ARs) in the residual prostatic tissue and bladder neck to relax the smooth muscle and alleviate post-TURP voiding symptoms. ${ }^{5}$ However, a-1 ARs are also expressed in the body of the urinary bladder, and the blockage of these receptors could cause the relaxation of the detrusor muscles. ${ }^{13}$ The upregulation of the expression of a-1 ARs in the presence of bladder outlet obstruction was observed by Bouchelouche et al. ${ }^{14}$; therefore, a-1 adrenergic antagonists may have a role in controlling post-TURP storage symptoms. Moreover, a recent study revealed that $\mathrm{a}-1$ adrenergic antagonists could increase the perfusion of the lower urinary tract and maximize bladder capacity, which could improve chronic ischemic injury due to prolonged bladder outlet obstruction, even after relief with TURP. ${ }^{15}$

A cohort study in Canada recruiting 58,000 patients revealed that $27 \%$ of the patients were under $\mathrm{a}-1$ adrenergic antagonists after a 90-day washout period after TURP. ${ }^{16}$ Although the rate of post-TURP a-1 adrenergic antagonist usage was lower than that in our study ( $27 \%$ vs. $41 \%)$, the absolute reduction rates of a-1 adrenergic antagonist usage before and after TURP were similar ( $35 \%$ and $42 \%$, respectively). Moreover, approximately one-third of the patients in the Canadian cohort study were prescribed with postTURP medications other than a-1 adrenergic antagonists, including 5a-reductase inhibitors, anticholinergics, or beta-3 agonists. Another retrospective study comparing the effects of different transurethral prostate procedures on medication discontinuation revealed a $74.4 \%$ reduction of $a-1$ adrenergic antagonist usage after TURP, which was higher than those observed in our study and the Canadian cohort study. ${ }^{17}$ The variable duration of bladder outlet obstruction before TURP and the extent of prostate resection may account for the differences in the discontinuation rates of a-1 adrenergic antagonists among these studies. The earlier the stage at which patients undergo TURP for bladder outlet obstruction, the higher the chance that bladder function could be preserved and, therefore, the lower the prevalence of post-TURP LUTS. The extent of prostate resection during TURP varies among urologists, and patients and should be balanced between the resolution of LUTS and development of surgical complications, such as ejaculation dysfunction, erectile dysfunction, and incontinence.

We found that a preoperative voiding efficiency of less than $75.48 \%$ is a predictive factor of continuous a-1 adrenergic antagonist usage after TURP. Similarly, a study involving the GreenLight laser vaporization of the prostate also reported that a preoperative voiding efficiency of greater than $75 \%$ is a predictor of surgery success. ${ }^{18}$ Patients with a preoperative voiding efficiency of greater than $75 \%$ had significantly higher improvements in the Qmax and International Prostate Symptom Scores than those whose voiding 
efficiency was less than $75 \%$. Voiding efficiency, compared with PVR, exhibits less intraindividual variation in predicting the severity of bladder outlet obstruction and capacity of bladder compensation in clinical settings. ${ }^{19}$ Other parameters, such as bladder outlet obstruction index (BOOI) and bladder contractility index $(\mathrm{BCl})$, could also help determine the bladder function in the presence of bladder outlet obstruction. Combining voiding efficiency with $\mathrm{BOOI}$ and $\mathrm{BCl}$ would be optimal for the evaluation of the lower urinary tract function of patients with $\mathrm{BPO} .{ }^{20}$ However, not every patient can undergo this painful, inconvenient, and invasive pressure flow examination to obtain $\mathrm{BOOI}$ and $\mathrm{BCI}$ before TURP. In the present study, we provided a simple and noninvasive parameter to predict the post-TURP a-1 adrenergic antagonist usage, which would be useful in preoperative evaluation and patient counseling.

Advanced age was also found to be associated with poor TURP outcomes in other studies. Campbell et al. found that advanced age could predict post-TURP usage of anticholinergics or beta-3 agonists. ${ }^{16}$ Another Italian cohort study revealed that the younger age was associated with better surgical outcomes. ${ }^{21}$ It is reasonable that the older the patients' age, the longer the duration of bladder outlet obstruction and the higher the possibility of bladder decompensation even after treatment with TURP. A similar trend was also found in our study, although it was not of statistical significance, which may be due to the small sample size.

The present study has a few limitations. First, this was a retrospective study. Some important clinical information was missing during data collection, such as preoperative urodynamic study or loss to followup postoperatively. Second, the sample size of this study was not large enough. Some possible predictive factors, such as age, were not of statistical significance. Third, the surgeries were not performed by a single urologist, and some operator-associated factors were difficult to identify. Nevertheless, a preoperative voiding efficiency of less than $75.48 \%$ remains not only a strong but also a simple and noninvasive predictive factor of post-TURP $\mathrm{a}-1$ adrenergic antagonist usage. A prospective study recruiting more patients undergoing TURP and other transurethral prostate procedures would help identify more preoperative predictors of post-TURP LUTS, which would be useful in preoperative evaluation and patient counseling.

\section{Conclusions}

Post-TURP LUTS requiring a-1 adrenergic antagonists for symptom control is not rare. A preoperative voiding efficiency of less than $75.48 \%$ is a simple and noninvasive predictive factor for this condition and would be helpful in preoperative evaluation and patient counseling.

\section{List Of Abbreviations}

ARs: adrenergic receptors

AUC: area under curve 
BPO: benign prostatic obstruction

BCl: bladder contractility index

BOOI: bladder outlet obstruction index

LUTS: lower urinary tract symptoms

Qmax: maximal flow rate

PVR: post-void residual urine

ROC: receiver operating characteristic

TURP: transurethral resection of the prostate

\section{Declarations}

Ethics approval and consent to participate: This study protocol is approved by the institutional review board (IRB) of our hospital (Taipei City Hospital) and is compliant with ethical standards. The IRB case number is "TCHIRB-11001002-E".

Consent for publication: The IRB of our hospital (Taipei City Hospital) approved the waiver of informed consent for this article. The data presented in this article is all de-identified.

Availability of data and materials: The data that support the findings of this study are available on request from the corresponding author, upon reasonable request. The data are not publicly available due to the privacy of the patients.

Competing interests: The authors declare no conflict of interest.

Funding: The authors received no financial support for the research, authorship, and publication of this article.

Authors' contributions: Wei-Ming Cheng conceived of the presented idea. Wei-Ming Cheng and Te-Wei Chang have been involved in writing the original manuscript and revising it critically for important intellectual content. Wei-Ming Cheng, Chan-Chi Chang, and Yi-Chun Chiu performed the majority of surgical intervention and followed up the patients. Yu-Hua Fan contributed to the final version of the manuscript. Che-Wei Hsu completed raw data collection. Shih-Han Weng assisted the statistical analyses and figure production.

Acknowledgments: We thank Prof. Hu for assistance with methodology.

\section{References}


1. Berry SJ, Coffey DS, Walsh PC, Ewing LL. The development of human benign prostatic hyperplasia with age. J Urol. 1984;132:474-9.

2. Haugen OA, Harbitz TB. Prostatic weight in elderly men. An analysis in an autopsy series. Acta Pathol Microbiol Scand A. 1972;80:769-77.

3. Moore RA. Benign hypertrophy of the prostate. A morphological study. J Urol. 1943;50:680-710.

4. Girman CJ, Jacobsen SJ, Tsukamoto T, Richard F, Garraway WM, Sagnier PP, et al. Health-related quality of life associated with lower urinary tract symptoms in four countries. Urology. 1998;51:42836.

5. Chughtai B, Simma-Chiang V, Kaplan SA. Evaluation and management of post-transurethral resection of the prostate lower urinary tract symptoms. Curr Urol Rep. 2014;15:434.

6. Mirone V, Imbimbo C, Longo N, Fusco F. The detrusor muscle: an innocent victim of bladder outlet obstruction. Eur Urol. 2007;51:57-66.

7. Steers WD. Pathophysiology of overactive bladder and urge urinary incontinence. Rev Urol. 2002;4 Suppl 4:S7-18.

8. Housami F, Abrams P. Persistent detrusor overactivity after transurethral resection of the prostate. Curr Urol Rep. 2008;9:284-90.

9. Kim SJ, Al Hussein Alawamlh O, Chughtai B, Lee RK. Lower urinary tract symptoms following transurethral resection of prostate. Curr Urol Rep. 2018;19:85.

10. Wada N, Watanabe M, Kita M, Matsumoto S, Kakizaki H. Analysis of bladder vascular resistance before and after prostatic surgery in patients with lower urinary tract symptoms suggestive of benign prostatic obstruction. Neurourol Urodyn. 2012;31:659-63.

11. Barbosa JABA, Reis ST, Nunes M, et al. The obstructed bladder: expression of collagen, matrix metalloproteinases, muscarinic receptors, and angiogenic and neurotrophic factors in patients with benign prostatic hyperplasia. Urology. 2017;106:167-72.

12. Kawabe K, Yoshida M, Homma Y, Silodosin Clinical Study Group. Silodosin, a new alpha1Aadrenoceptor-selective antagonist for treating benign prostatic hyperplasia: results of a phase III randomized, placebo-controlled, double-blind study in Japanese men. BJU Int. 2006;98:1019-24.

13. Malloy BJ, Price DT, Price RR, Bienstock AM, Dole MK, Funk BL, et al. Alpha1-adrenergic receptor subtypes in human detrusor. J Urol. 1998;160:937-43.

14. Bouchelouche K, Andersen L, Alvarez S, Nordling J, Bouchelouche P. Increased contractile response to phenylephrine in detrusor of patients with bladder outlet obstruction: effect of the alpha1 $\mathrm{A}$ and alpha1D-adrenergic receptor antagonist tamsulosin. J Urol. 2005;173:657-61.

15. Pinggera GM, Mitterberger M, Pallwein L, Schuster A, Herwig R, Frauscher F, et al. Alpha-blockers improve chronic ischaemia of the lower urinary tract in patients with lower urinary tract symptoms. BJU Int. 2008;101:319-24.

16. Campbell J, Reid J, Ordon M, Welk B. The utilization of benign prostatic hyperplasia and bladderrelated medications after a transurethral prostatectomy. Urology. 2019;130:126-31. 
17. Sabharwal NC, Shoskes DA, Dielubanza EJ, Ulchaker JC, Fareed K, Gill BC. Comparative effectiveness of transurethral prostate procedures at enabling urologic medication discontinuation: a retrospective analysis. Urology. 2019;134:192-8.

18. Choo MS, Cho SY, Han JH, Lee SH, Paick J-S, Son H. The cutoff value of bladder voiding efficiency for predicting surgical outcomes after GreenLight HPS ${ }^{T M}$ laser photoselective vaporization of the prostate. J Endourol. 2014;28:969-74.

19. $\mathrm{Ku} \mathrm{JH}, \mathrm{Cho}$ SY, Oh SJ. Residual fraction as a parameter to predict bladder outlet obstruction in men with lower urinary tract symptoms. Int J Urol. 2009;16:739-44.

20. Abrams $P$. Bladder outlet obstruction index, bladder contractility index and bladder voiding efficiency: three simple indices to define bladder voiding function. BJU Int. 1999;84:14-5.

21. DE Nunzio, Lombardo R, Nacchia A, Deroma M, Alkhatatbeh $H$, Brassetti A, et al. Young Academic Urologists' benign prostatic obstruction nomogram predicts clinical outcome in patients treated with transurethral resection of prostate: an Italian cohort study. Minerva Urol Nefrol. 2018;70:211-7.

\section{Figures}




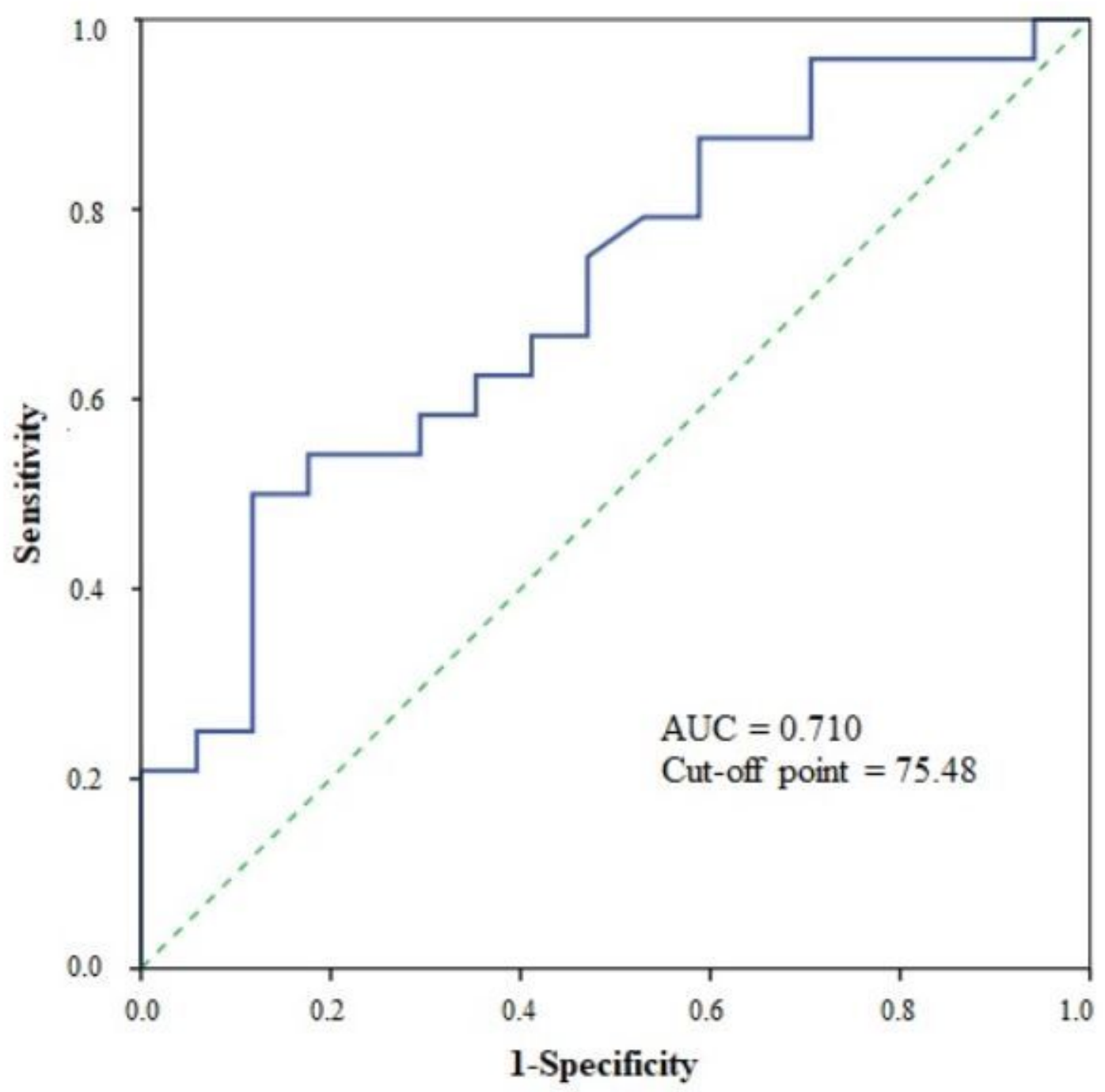

Figure 1

Receiver operating characteristic curve of voiding efficiency. This is used to predict the long-term usage of a-1 adrenergic antagonists after transurethral resection of the prostate 